Powell, E. O. (1956). J. gen. Microbiol. 15, 492-511

\title{
Growth Rate and Generation Time of Bacteria, with Special Reference to Continuous Culture
}

\author{
By E. O. POWELL
}

Microbiological Research Establishment, Ministry of Supply, Porton, Wiltshire

SUMMARY: The relations between growth rate, generation time distribution and age distribution in growing bacterial cultures are derived. The effect of inheritance on generation time is probably negligible. Some applications to experimental data exemplify the mathematical results. The validity of the principal assumptions is discussed.

It appears to be tacitly assumed by many bacteriologists, in particular by some who are concerned with the study of continuous cultures, that there is a simple relationship between growth rate and generation time. It is sometimes stated that during the phase of steady exponential growth of a batch culture the following equation holds:

$$
\frac{d \log N}{d t}=\nu=\frac{\log 2}{\bar{\tau}},
$$

where $N$ (supposed very large) is the number of organisms in the culture, $\nu$ is the number growth rate constant, and $\bar{\tau}$ is the mean generation time (in a sense not clearly defined). Equation (1) is false except in special cases that are apparently never realized in practice. Similarly, in the steady state of a continuous culture running at dilution rate $D$ it is usually false that

$$
D=\frac{\log 2}{\bar{\tau}} \text {. }
$$

The true relationships, though more subtle, differ quantitatively from (1) and (2) by very little, but so far as (2) is concerned, that little is an expression of the selective power which is so important a feature of continuous culture.

In this paper. I describe some simple quantitative features of bacterial populations which result from the dispersion of the individual generation times. I assume (i) that the populations under discussion are homogeneous, i.e. that they consist of organisms of only one type, and that the cultures have continued in steady growth long enough for the age distribution to have become constant; (ii) that the numbers of organisms present are so large that variations can be regarded as continuous, and statistical fluctuation as negligible. Some of the mathematical results are not new, but they are somewhat inaccessible to bacteriologists, and I have given simple derivations, glossing over many difficulties which the pure mathematician must attend to, but which are resolved by observation for the bacteriologist.

To avoid the use of inappropriate or ambiguous phrases, I adopt the following terminology: the 'inception' and 'termination' of an organism are 
respectively the events by which it becomes a recognizably separate entity (by fission of its parent) and by which it ceases to be so (by itself dividing). The same words may be used to mean the epochs of these events. The 'age' of an organism (to be distinguished from the age of a culture) is then the time which has elapsed since its inception; a 'young' organism is one whose age is small compared with the mean generation time.

\section{Mass and number grozeth rate}

If in a batch culture $x$ and $n$ are the mass and number of organisms, respectively, per unit volume, we can write for the phase of 'logarithmic' growth

$$
\begin{aligned}
& \frac{d x}{d t}=\mu x, \\
& \frac{d n}{d t}=\nu n,
\end{aligned}
$$

where $\mu$ and $\nu$ are the mass and number growth rates. If the population density is not too large, so that the organisms are well nourished, $\mu$ and $\nu$ take constant values $\mu_{m}$ and $v_{m}$ which are characteristic of the medium. In a well-balanced medium, thoroughly aerated, it appears possible to grow some organisms at a constant rate for about thirty generations, i.e. until the inoculum has multiplied $10^{\circ}$-fold. Thus Powell (1956a) from observations on Aerobacter cloacae $\dagger$ growing on a virtually unlimited medium under the microscope, found the growth rate to be $0.0143 \mathrm{~min}^{-1}$; Dr D. Herbert (private communication), working with the same organism and medium on a larger scale, found the growth rate to be sensibly the same up to a population density of $c .10^{10} / \mathrm{ml}$.

Clearly, $\mu$ and $\nu$ must have the same value during steady growth, since otherwise the size of the organisms would increase or decrease indefinitely.

The mass growth rate may be written as a function of nutrilite concentration. In the simplest case, when growth is limited by a deficiency of a single substance, all others being present in excess, we have approximately

$$
\mu=\mu_{m} \frac{s}{\bar{K}+s}
$$

where $s$ is the concentration of limiting nutrilite, and $K$ is a constant, the 'saturation constant'. Usually $K$ is exceedingly small, so that $\mu \bumpeq \mu_{m}$ except when $s$ is of the same order of magnitude as $K$, or less (cf. Monod, 1942; Dagley \& Hinshelwood, 1938). In the experiments already quoted, Herbert found that growth and oxygen uptake ceased quite abruptly when the medium was exhausted of glucose. Although (4) has no real theoretical basis, it agrees with observation so far as present experimental techniques can determine.

† This organism (NCTC 8197) was formerly given the specific name aerogenes by the National Collection of Type Cultures. The same strain was called Bacterium aerogenes in Powell (1955) and Aerobacter aerogenes in Powell (1956a). 
Provided, then, that the culture is adequately nourished, we can write for $(\mathbf{3} b)$

or

$$
\begin{gathered}
\frac{d n}{d t}=\nu_{m} n, \\
n=n_{0} e^{\nu_{m}\left(t-t_{0}\right)}
\end{gathered}
$$

where $n_{0}$ is the (numbe:) concentration at time $t_{0}$. The growth may be characterized by a quantity $\bar{\tau}_{e}$, the 'doubling time' or 'mean effective generation time', instead of by $v_{m}$; this is the time required for the number to double:

$$
\begin{gathered}
2 n_{0}=n_{0} e^{\nu^{\tau} e^{\tau}}, \\
\bar{\tau}_{e}=\frac{\log 2}{v_{m}} .
\end{gathered}
$$

(It is sometimes convenient to distinguish a mass 'doubling time', $\bar{\tau}_{d}$ (cf. Herbert, Elsworth \& Telling, 1956) related to $x$ as $\bar{\tau}_{e}$ is to $n$. The reciprocals of $\bar{\tau}_{e}$ and $\bar{\tau}_{d}$ are called 'taux de naissance' or 'taux de croissance' by French authors.)

\section{The distribution of generation times}

The generation times of individual organisms are far from uniform; the scatter appears to depend on the growth medium, and is on the whole greater in multicellular than in unicellular species (Powell, 1955, 1956b). Examples are:

(i) (Powell, 1955.) Bacillus mycoides growing on tryptic meat broth at $35^{\circ}$ :

$\begin{array}{lc}\text { Estimated mean generation time } & 28.7 \mathrm{~min} . \\ \text { Estimated standard deviation } & 14.2 \mathrm{~min} . \\ \text { Coefficient of variation } & 0.496\end{array}$

(ii) (Powell, 1956b.) Streptococcus faecalis growing on tryptic meat broth at $35^{\circ}$ :

$\begin{array}{lc}\text { Estimated mean generation time } & 26 \cdot 1 \mathrm{~min} . \\ \text { Estimated standard deviation } & 3.5 \mathrm{~min} . \\ \text { Coefficient of variation } & 0 \cdot 133\end{array}$

Some care is needed in defining the distribution of generation time, especially when actual measurements are envisaged (Powell, 1955). The frequency function of generation time, $f(\tau) d \tau$, is the probability that a newly formed organism will have a generation time in the range $\tau, \tau+d \tau$. This suffices to define the distribution provided that the particular value of $\tau$ is independent of the ancestry of the organism; I shall make the assumption in this and the next two sections. In all known examples, $f(\tau)$ is a positively skew curve, usually leptokurtic, and with no evidence of multimodality. Various functional forms have been suggested for it (Rahn, 1932; Kendall, 1948; Kendall \& Waugh, cited in Kendall, 1952 ; Powell, 1956 b). Of these the Pearson type III distribution is the most convenient, and usually fits adequately:

$$
f(\tau)=\frac{\tau^{g-1} e^{-\tau / m}}{m^{g} \Gamma(g)}
$$




\section{Growth rate and generation time of bacteria}

The parameters $g, m$ are simply related to well-known statistics:

Arithmetic mean of $\boldsymbol{\tau}=\mathbf{a}=\mathbf{g m}$,

Coefficient of variation of $\tau=c=g^{-\frac{1}{2}}$.

Age distribution and growth rate in batch culture

The age distribution in a growing culture has a curious and interesting property which is not generally known; roughly speaking, the youngest organisms are present in greatest number.

To discover the distribution, write $\phi(a)$ for the frequency function of age and consider a culture containing a large number $N$ of organisms at time $t=0$, say. There are $N \phi(a) d a$ organisms whose ages are in the range $a, a+d a$. Now write

$$
F_{-}(\tau)=\int_{\tau}^{\infty} f(\xi) d \xi
$$

for the proportion of organisms whose generation times are greater than $\tau$. It can then be seen that if an organism has already attained the age $a$, its chance of attaining the greater age $a+t$ without dividing is

$$
\frac{F_{-}(a+t)}{F_{-}(a)} \text {. }
$$

Hence of the original $N \phi(a) d a$ organisms,

$$
\frac{N \phi(a) F_{-}(a+t) d a}{F_{-}(a)}
$$

are still extant at time $t$. But meanwhile the culture has grown, and now contains $N e^{\nu} m^{l}$ organisms, instead of $N$. The survivors then form a fraction

$$
\frac{\phi(a) F_{-}(a+t) e^{-\nu_{m} l} d a}{F_{-}(a)}
$$

of the whole number, and their ages now lie in the range $a+t, a+t+d a$. Since the culture is by hypothesis in a steady state this fraction must be $\phi(a+t) d a$. Thus the difference equation

$$
\frac{\phi(a) F_{-}(a+t) e^{-\nu_{m} t}}{F_{-}(a)}=\phi(a+t)
$$

is to hold for all $t$. In particular, for very small $t$

$$
\begin{aligned}
F_{-}(a+t) & =t d F_{-} / d a+F_{-}(a), \\
\phi(a+t) & =t d \phi(a) / d a+\phi(a), \\
e^{-\nu_{m}} & =1-\nu_{m} t,
\end{aligned}
$$

neglecting powers of $t$ above the first. Hence, substituting in (6)

$$
\begin{gathered}
\frac{1}{F_{-}(a)} \frac{d}{d a}\left\{F_{-}(a)\right\}-\nu_{m}=\frac{1}{\phi(a)} \frac{d}{d a}\{\phi(a)\}, \\
\phi(a)=\phi(0) e^{-\nu_{m} a} F_{-}(a)=\phi(0) e^{-\nu_{m} a} \int_{a}^{\infty} f(\tau) d \tau,
\end{gathered}
$$


where $\phi(0)$ is a constant, yet to be determined, which makes

$$
\int_{0}^{\infty} \phi(a) d a=1 \text {. }
$$

The exponential function and the integral on the right of $(7)$ are both positive, and decrease steadily as $a$ increases, hence $\phi(a)$ does so: the age distribution has a maximum at $a=0$. To complete this investigation we have to determine $\phi(0)$ and the growth-rate constant $\nu_{m}$ in terms of $f(\tau)$.

Since each fission increases the number of organisms by one, the (absolute) fission rate divided by the number of organisms present is the growth rate. The chance that an organism will attain an age $a$ at least is $F_{-}(a)$; the chance that it will attain age $a+d a$ at least is $F_{-}(a+d a)$,

$$
=F_{-}(a)-f(a) d a \text {; }
$$

among organisms which have attained age $a$ the fraction $f(a) d a / F_{-}(a)$ terminate during the succeeding short interval $d a$; the fission rate among such organisms is $f(a) / F_{-}(a)$. There are at any given time say $N$ organisms in the culture, of which $N \phi(a)$ are of age $a$. These contribute

$$
N \phi(a) f(a) d a / F_{-}(a)
$$

to the fission rate. Taking into account organisms of all ages,

$$
\begin{aligned}
\nu_{m} & =\frac{1}{N} \int_{0}^{\infty} \frac{N \phi(a) f(a) d a}{F_{-}(a)} \\
& =\int_{0}^{\infty} \phi(0) e^{-\nu_{m} a} f(a) d a .
\end{aligned}
$$

Now integrate (7) by parts between limits 0 and $\infty$ :

$$
\int_{0}^{\infty} \phi(a) d a=1=\phi(0)\left[\frac{e^{-\nu_{m} a}}{-\nu_{m}} F_{-}(a)\right]_{0}^{\infty}-\phi(0) \int_{0}^{\infty} \frac{e^{-\nu_{m} a}}{\nu_{m}} f(a) d a .
$$

Here the last term is unity, by $(8)$, and $F_{-}(0)=1$. Hence

$$
\phi(0)=2 v_{m} \text {. }
$$

Finally, we may write for the age distribution, instead of (7).

$$
\phi(a)=2 \nu_{m} e^{-\nu_{m} a} \int_{a}^{\infty} f(\tau) d \tau,
$$

while the growth rate cannot be written explicitly, but must be determined (using (8) again) by

$$
2 \int_{0}^{\infty} e^{-\nu_{m} \tau} f(\tau) d \tau=1
$$

(cf. Harris, 1951). It is evident that $\nu_{m}$ cannot equal $(\log 2) / \mathbf{a}$ in general, though in fact the difference is quite small for unicellular organisms, the dispersion of whose generation times is moderate.

When the generation time is not dispersed, we have $\tau=\mathbf{a}$ for every organism and $f(\tau)=0$ except at $\tau=\mathbf{a}$. Also, by $(10), v_{m}=(\log 2 / \mathbf{a})$. Then,

$$
\begin{aligned}
F_{-}(a) & =1 & & (a<\mathbf{a}) \\
& =0 & & (a>\mathbf{a}),
\end{aligned}
$$


and

$$
\begin{aligned}
\phi(a) & =2 \nu_{m} e^{-\nu_{m} a} \quad(a<\mathbf{a}) \\
& =0 \quad(a>\mathbf{a}) \\
\phi(\mathrm{a}-0) & =\nu_{m}=\frac{1}{2} \phi(0) .
\end{aligned}
$$

The last equation also follows from the consideration that the number of organisms which are just about to divide must be half the number just newly formed. This special case is illustrated in Fig. 1 (curve (i)). The distributions actually found are smoothed-out versions of it; the sharpness of the fall in $\phi(a)$ near $a=\mathrm{a}$ is less, the greater the dispersion of $\tau$ (curves (ii), (iii); see also Fig. $4 a, b)$.

The expression $2 e^{-\nu_{m} \tau} f(\tau)$ is everywhere positive, and so by (10) it is a frequency function. It has an important significance, more especially in continuous cultures, and it is therefore convenient to give it a distinctive name and symbol; I call it the 'carrier distribution', $\mathscr{C}(\tau)$. We have already seen that of the $N \nu_{m}$ fissions per unit time occurring in the culture, $N \phi(a) f(a) d a / F_{-}(a)$ are the terminations of organisms which reach the age $a$ but fail to reach $a+d a$, i.e. of those whose generation times $\tau$ lie in this interval. The distribution of the generation times of those organisms actually extant at any given time is $f(\tau)$, but the distribution of generation times of their immediate ancestors (mothers) is

$$
\frac{N \phi(\tau) f(\tau) d \tau}{N \nu_{m} F_{-}(\tau)}=2 e^{-\nu_{m} \tau} f(\tau) d \tau=\mathscr{C}(\tau) d \tau .
$$

A difficulty arises if the culture throws off some non-viable organisms. Suppose that the constant fraction $\alpha$ of newly formed organisms are viable in the sense that they terminate within a finite time. Then the generation time distribution is such that

$$
\int_{0}^{\rightarrow \infty} f(\tau) d \tau=\alpha,
$$

and in addition there is a singular component contributing $1-\alpha$ to the total frequency at $\tau=\infty$. It is not clear that viable organisms can be certainly distinguished from non-viable, but adequate conventional standards can be adopted in given circumstances. We can then if we wish define a frequency function $f^{*}(\tau)$ for the generation times of viable organisms only. If $\alpha<1$, the mean of $f(\tau)$ will be infinite; that of $f^{*}(\tau)$ may or may not be. Obviously for finite $\tau, \alpha f^{*}(\tau)=f(\tau)$ and we can write instead of (10)

$$
1=2 \alpha \int_{0}^{\infty} e^{-\nu_{m} \tau} f^{*}(\tau) d \tau
$$

Even if the mean of $f^{*}(\tau)$ or $f(\tau)$ is infinite, the integral (10) converges and gives a finite non-zero growth rate if $\alpha>\frac{1}{2}$. We have no right to expect that the mean is finite even in $f^{*}(\tau)$, therefore. The relatively few measurements that have been made do not in fact suggest any unusual instability in sample means such as would be symptomatic of an infinite population mean. For $\alpha=\frac{1}{2}$ we have

$$
1=\int_{0}^{\infty} e^{-v_{m} \tau} f^{*}(\tau) d \tau=\int_{0}^{\infty} f^{*}(\tau) d \tau
$$


and $v_{m}$ must be zero. For smaller $\alpha, v_{m}$ will be negative, i.e. the culture will die out. This conclusion is more readily reached by other methods (e.g. Feller, 1950). In my experience, $\alpha>0.99$ for well-nourished organisms (Powell, $1956 b$ ), and I think it may usually be assumed that $\alpha=1$ in batch cultures; it is easy to make a correction where it is necessary and practicable to distinguish living organisms from dead.

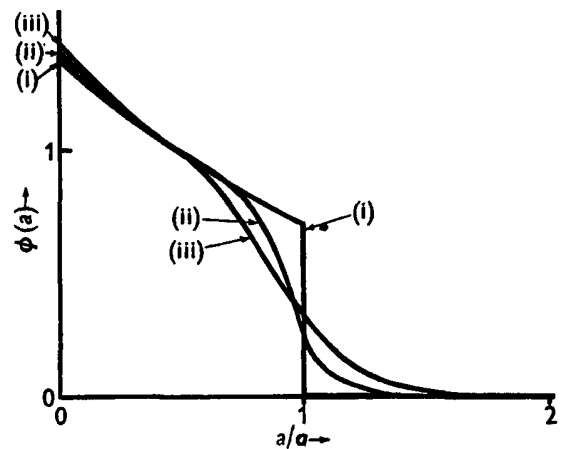

Fig. 1

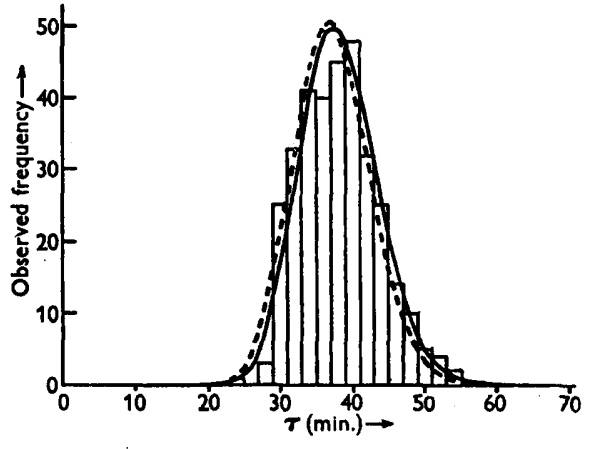

Fig. 2

Fig. 1. Effect of dispersion of $\tau$ on age distribution, schematic. (i) No dispersion ; (ii) $f(\tau)$ a Pearson Type III with $g=49(c=0 \cdot 14)$; (iii) $f(\tau)$ a Pearson Type III with $g=16$ $(c=0 \cdot 25)$.

Fig. 2. A generation time distribution of Pseudomonas aeruginosa. Histogram: observed frequencies. Continuous curve: Pearson Type III distribution of best fit. Dotted curve : carrier distribution.

\section{Examples}

(i) (Data from Powell, 1956 b.) Pseudomonas aeruginosa growing in a defined medium containing glucose, glutamic acid, succinic acid, thiosulphate and the usual salts, at $35^{\circ}$ :

$$
\begin{aligned}
\mathbf{a} & =\mathbf{3 8 . 1} \mathrm{min} ., \\
\operatorname{var} \tau & =\mathbf{2 8 . 7}, \\
c & =\mathbf{0} \cdot 141 .
\end{aligned}
$$

The distribution was well represented by a Pearson Type III (eqn. 5), with parameters $g=50 \cdot 6, m=0.753$ (Fig. 2). The growth rate was estimated in two ways :

(a) From eqn. (10) by insertion of (5):

$$
\begin{aligned}
1 & =2 \int_{0}^{\infty} \frac{e^{-v_{m} \tau} \tau^{g-1} e^{-\tau / m} d \tau}{m^{\sigma} \Gamma(g)} \\
& =2\left(1+m \nu_{m}\right)^{-\sigma} ; \\
\nu_{m} & =\left(2^{1 / g}-1\right) / m .
\end{aligned}
$$

(b) By replacing the integral in (10) by a finite sum over the observed frequencies, using trial values of $\nu_{m}$, and interpolating:

(a) gave $\nu_{m}=0.018314$,

(b) gave $\nu_{m}=0.018314$, 
while $(\log 2) / a=0.018189-a$ difference of $0.7 \%$ only. The carrier distribution, calculated from

$$
\begin{aligned}
\mathscr{C}(\tau) & =\frac{2 e^{-\nu_{m} \tau} \tau^{g-1} e^{-\tau / m}}{m^{\rho} \Gamma(g)} \\
& =\frac{2 \tau^{g-1}}{m^{g} \Gamma(g)} \exp \left(-\frac{\tau}{m} 2^{1 / g}\right)
\end{aligned}
$$

(again a Pearson Type III) is also shown in Fig. 2.

(ii) (New data.) Bacillus megaterium growing on peptone water at $\mathbf{3 5}^{\circ}$. Observations were begun at $2.5 \mathrm{hr}$. after inoculation of spores, and were continued for $80 \mathrm{~min}$. in each experiment. The experiments and reduction of the data were conducted as described in Powell (1955) for $B$. subtilis and $B$. mycoides. The sample of generation times obtained from experiments restricted to a fixed period is systematically biased, and on the assumption that the true $f(\tau)$ is of Pearson Type III, the frequency function to be fitted to the observations is (apart from a normalizing factor)

$$
\left\{e^{\nu_{m}(80-\tau)}-1\right\} \tau^{g-1} e^{-\tau / m} \quad(\tau \leqslant 80) .
$$

This curve was fitted by the method of moments (Fig. $3 a$ ), and the test for goodness of fit gave $\chi_{12}^{2}=18 \cdot 25 ; P\left(\chi^{2}\right)=0 \cdot 11$. The estimates of the parameters were

whence

$$
\begin{aligned}
g & =8 \cdot 26, \\
m & =2 \cdot 70 ; \\
g^{-\frac{1}{2}} & =c=0 \cdot 348, \\
m g & =\mathbf{a}=22 \cdot 3 \mathrm{~min} ., \\
(\log 2) / \mathbf{a} & =0.03113 \mathrm{~min} .^{-1}, \\
v_{m} & =0.03247 \mathrm{~min} .^{-1} .
\end{aligned}
$$

The greater difference of the last two figures (c. $4.2 \%$ ), as compared with the previous example, reflects the greater dispersion of $\tau$. From the experimental data a growth curve was constructed by adding together the number of organisms under observation at corresponding times in each experiment. It was satisfactorily linear (Fig. $3 b$ ), and gave another estimate of the growth rate constant

$$
v_{m}=\mathbf{0} \cdot 03259 \text {, }
$$

in fair agreement with that calculated from the frequency function.

At the end of each experiment, the ages of the organisms then present were recorded, so that the age-distribution could be built up. The observed distribution was compared with that calculated from $f(\tau)$ :

$$
\phi(a)=2 \nu_{m} e^{-\nu_{m} a} I_{-}(a / m, g),
$$

where $I_{-}(a / m, g)$ is the incomplete gamma function

$$
\frac{1}{\Gamma(g)} \int_{a / m}^{\infty} \xi^{g-1} e^{-\xi} d \xi .
$$

(A convenient table of this function is given in Pearson \& Hartley (1954). They actually tabulate $P\left(\chi^{2}, \nu\right): I_{-}(a / m, g)=P(2 a / m, 2 g)$.) The histogram 
(Fig. 4a) reproduces the general features of $\phi(a)$, but the fit is very bad, mainly because of the excess of observations in the cell $a=16$. This excess, in spite of its improbability, can only be ascribed to accident. There are two factors which increase the expectation of $\chi^{2}$ in experiments of this kind: (i) there is high correlation between the generation times of sister organisms, so most observations of age occur in pairs, and the variance in each cell is nearly doubled; (ii) errors of judgement in estimating times of fission result in observations being assigned to cells adjacent to the true ones, not at random.
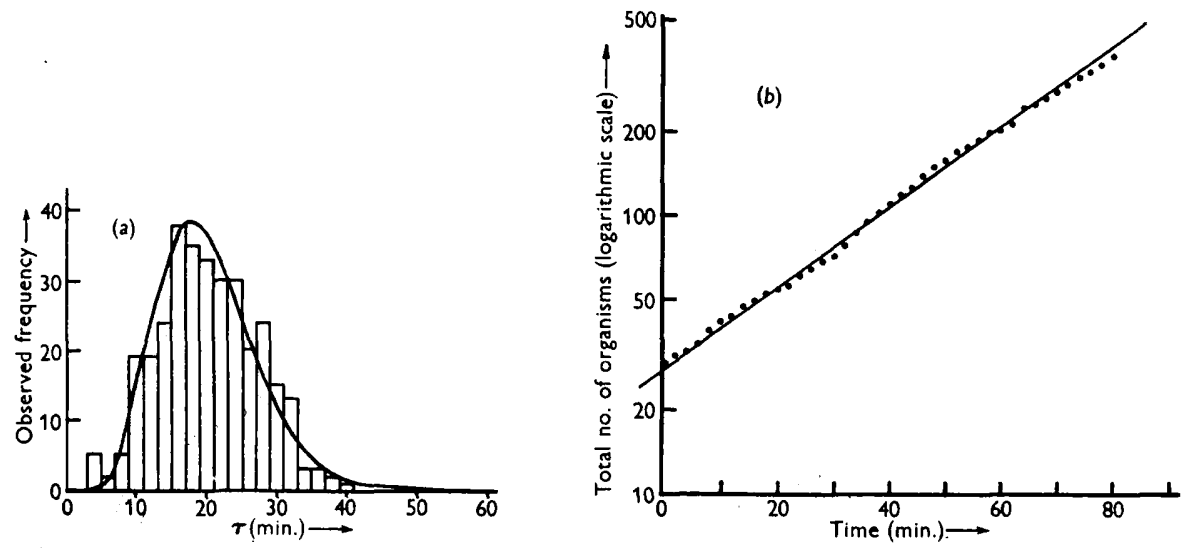

Fig. 3. (a) A generation time distribution of Bacillus megaterium. Histogram: observed frequencies. Continuous curve: modified Pearson Type III distribution. (b) Growth curve of $B$. megaterium under the same conditions as $(a)$.

The median age (from either the observed or calculated distribution) is about 7 min., i.e. in a sample from such a culture half the organisms would be less than $7 \mathrm{~min}$. old.

(iii) (Data from Powell, 1955.) Bacillus mycoides growing on tryptic meat broth at $35^{\circ}$. The estimated parameters of the Pearson Type III distribution were:

whence

$$
\begin{aligned}
g & =4.07, \\
m & =7 \cdot 04, \\
c & =0.496, \\
\mathrm{a} & =28.7 \mathrm{~min} ., \\
(\log 2) / \mathbf{a} & =0.02419 \mathrm{~min} .^{-1}, \\
\nu_{m} & =0.02637 \mathrm{~min} .^{-1} .
\end{aligned}
$$

The observed and calculated age distribution are compared in Fig. $4 b$; the fit is fairly $\operatorname{good}\left(\chi_{18}^{2}=21 \cdot 58 ; P\left(\chi^{2}\right)=0 \cdot 25\right)$. The median is about $11 \mathrm{~min}$.

\section{Age distribution and growth rate in continuous culture}

In a continuous culture maintained in a steady state, the growth rate is equal to the dilution rate, $D$. ( $D$ is the fraction of the volume of culture displaced by the inflowing medium, per unit time.) The generation time distribution is therefore dependent on $D$, and we may now write it as $f_{D}(\tau)$. 
The immediate determinant of the growth rate is the concentration of the medium (or one or more constituents of it) according to some such law as (4):

$$
\nu=\mu=D=\mu_{m} \frac{s}{K+s} .
$$

This is true of all types of continuous culture. For simplicity, consider the case where the growth rate is restricted, if it is restricted at all, by deficiency of a single nutrilite $(s)$, and suppose that to produce $Y \mathrm{~g}$. of organisms, $1 \mathrm{~g}$. of this nutrilite must be consumed ( $Y$ is called the yield constant.). Then,
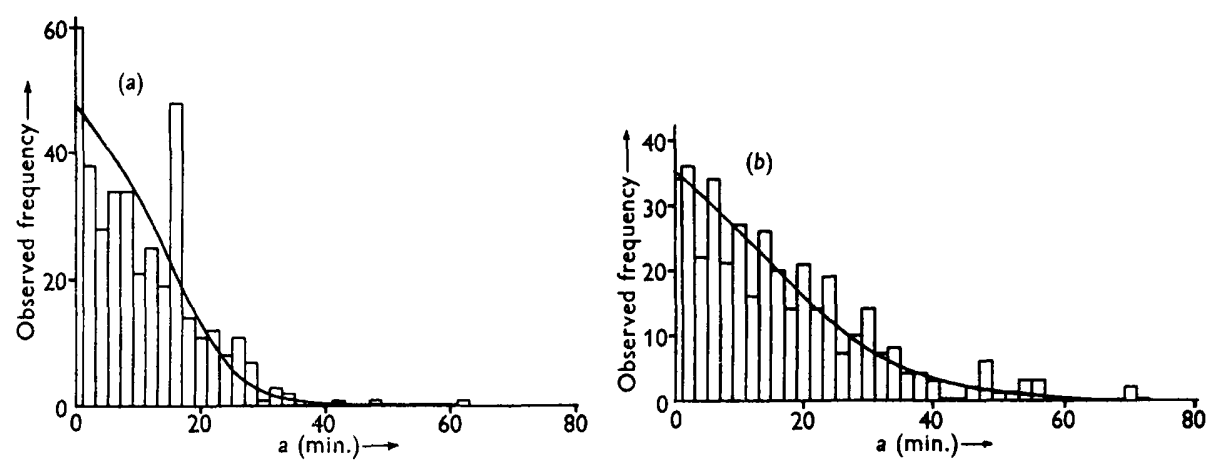

Fig. 4. (a) Age distribution of $B$. megaterium in steady growth. Histogram: observed frequencies. Continuous curve: calculated from the fitted distribution of Fig. $3 a$. (b) Age distribution of $B$. mycoides in steady growth. Histogram : observed frequencies. Continuous curve: calculated from the generation time distribution.

if $x$ is the mass concentration of organisms and $s$ the concentration of limiting nutrilite, the following equation holds at all points in the system (including medium reservoirs and receivers):

$$
s_{R}=s+x / Y,
$$

where $s_{R}$ is the nutrilite concentration in the inflowing medium. In the 'chemostat' (Novick \& Szilard, 1950) or Monod (1950) types of apparatus, $D$ is fixed by the experimenter, and the concentration of organisms adjusts itself to satisfy (13) and (14):

$$
x=Y\left(s_{R}-s\right)=Y\left(s_{R}-\frac{K D}{\mu_{m}-D}\right) ;
$$

it can do so only if $D<\mu_{m} s_{R} /\left(K+s_{R}\right)$. In the 'turbidostat' (Bryson \& Szybalski, 1952) type, the experimenter fixes $x$, and $D$ adjusts itself to satisfy the same conditions. Here the limitation is that $x<s_{R} Y$. Novick's (1955) insistence on an essential difference between the two systems is a mistake; they have different useful ranges of applicability, but the relation of organisms to environment is the same for both, and they can be analysed in the same way up to the point at which experimental control is in question. They will not, however, behave in the same way during changes of the working conditions.

For the time being I shall assume that the mixing in the culture vessel is 'perfect', i.e. that the ingoing medium is instantaneously and homogeneously 
dispersed throughout the working volume, and that the effluent is drawn equally from all points of the vessel. Then, if an organism is present at time $t$, the chance of its remaining in the vessel until the later time $t_{1}$, at least, is $\exp \left\{-D\left(t_{1}-t\right)\right\}$.

All the frequency functions which have been proposed to represent the distribution of generation times can be written in the form

$$
f\left(\tau / m ; g_{1}, g_{2}, \ldots\right) d(\tau / m)
$$

where the parameters $g_{r}$ are independent of $m$. Changes in $m$ alter the timescale only, and all the moments of the curves can be written as $m^{s} \boldsymbol{M}_{s}\left(g_{1}, g_{2}, \ldots\right)$. If generation times are determined by a mechanism of the Kendall (1948, 1952) or more general Kendall \& Waugh type (Kendall, 1952), we might expect only the scale of the distribution to be altered under conditions of moderately restricted growth. No detailed observations have yet been made under such conditions, but it will be seen that the same principles as apply to batch culture apply here. The only change is that the growth rate $\nu$ is now not $\nu_{m}$ (in general) and is equal to $D$. As a reminder of the dependence on $D$, it is convenient to write $\phi_{D}(a), F_{D-}(\tau)$, as well as $f_{D}(\tau)$, where before we had $\phi(a), \boldsymbol{F}_{-}(\tau)$.

Suppose a culture vessel contains $N$ organisms (by hypothesis constant). At time $t=0$, say, there are $N \phi_{D}(a) d a$ organisms whose ages lie in the range $a, a+d a$. Of this number, at time $t$,

$$
\frac{N \phi_{D}(a) F_{D-}(a+t) d a}{F_{D-}(a)}
$$

will still not have divided, but of the survivors only the fraction $e^{-D t}$ will still be in the vessel; the rest will have been washed out. Hence

$$
\frac{\phi_{D}(a) F_{D-}(a+t) e^{-D t}}{F_{D_{-}}(a)}=\phi_{D}(a+t)
$$

and, by the same reasoning as was previously used,

$$
\phi_{D}(a)=\phi_{D}(0) e^{-D a} \int_{a}^{\infty} f_{D}(\tau) d \tau,
$$

where $f_{D}(\tau)$ is the generation time distribution under the conditions obtaining in the culture vessel.

The relation between growth rate and generation time is more simply obtained than in batch culture. The chance that an organism chosen at random will terminate in the vessel must be $\frac{1}{2}$. For if it terminates in the vessel, it produces two daughters; if it does not, it produces none; and the expected number of its immediate progeny must be 1 to keep $N$ constant. The chance that its generation time is in the range $\tau, \tau+d \tau$ is $f_{D}(\tau) d \tau$; the chance that it remains in the vessel until it terminates is $e^{-D \tau}$. Thus we have

$$
\frac{1}{2}=\int_{0}^{\infty} e^{-D \tau} f_{D}(\tau) d \tau
$$

formally the same as (10). It follows that $\phi_{D}(0)$ in $(15)$ is $2 D$.

We now have a carrier distribution

$$
\mathscr{C}_{D}(\tau)=2 e^{-D \tau} f_{D}(\tau)
$$


it is not difficult to see that $\mathscr{C}_{D}(\tau)$ is the generation time distribution of those organisms which remain in the vessel until their termination, i.e. of those which are responsible for the maintenance of the culture.

Equation (17) shows that a continuous culture discriminates heavily against organisms of unusually long generation time. As can be seen from Fig. 2, there is little difference between $\mathscr{C}_{D}(\tau)$ and $f_{D}(\tau)$ for moderate values of $\tau$ if the dispersion is not great, but

$$
\mathscr{C}_{D}(\tau) / f_{D}(\tau)=2 e^{-D \tau} \rightarrow 0 \quad \text { as } \quad \tau \rightarrow \infty
$$

Of course, by (11) the same discrimination operates in batch cultures, but the outcome is greatly complicated by the accelerations of growth which occur at the beginning and end of the 'logarithmic' phase. In continuous cultures, we should expect the population to become very quickly stable and homogeneous; organisms of large $\tau$, which contribute little to the growth rate, are eliminated altogether from the system. Conversely, the growth rate is affected by any persistent correlation between the generation times of related organisms (e.g. between mothers and daughters). Or, if the growth rate is fixed, the composition of the population must adjust itself. The effect of correlation is considered in the next section.

I hope to deal elsewhere with the selective property of a continuous culture as it affects the growth of contaminants and mutants.

Conditions of restricted growth such as occur when $D$ is much less than $\nu_{m}$ appear to cause the death of an appreciable proportion of the organisms (Novick, 1955; Powell, 1956a); it cannot then be assumed that the index of viability, $\alpha$, is either constant or near unity. In the steady growth of a batch culture, it can be shown that the proportion of viable organisms $(V)$ is $2 \alpha-1$ (Topley \& Wilson's Principles, 1955; the 'generation index', $p$, which they use is $2 \alpha$ ). The same ratio obtains in continuous cultures: Suppose the culture contains a constant number, $N$, of organisms of which $N V$ are viable and $N(1-V)$ non-viable. During a short interval $d t, D N d t$ fissions occur, and with them are associated a loss of $D N d t$ viable organisms, a gain of $2 \alpha D N d t$ viable organisms and a gain of $2(1-\alpha) D N d t$ non-viable. At the same time $D N d t$ organisms are removed by the flow through the vessel; of these $D N V d t$ are viable and $D N(1-V) d t$ non-viable. Hence in the steady state

and

$$
\begin{aligned}
& -D N d t+2 D N \alpha d t-D N V d t=0, \\
& 2(1-\alpha) D N d t-D N(1-V) d t=0,
\end{aligned}
$$

$$
\begin{gathered}
V=2 \alpha-1, \\
1-V_{/}=2(1-\alpha),
\end{gathered}
$$

as asserted. If $f_{D}^{*}(\tau)$ is the generation time distribution for viable organisms only, we have

$$
\begin{aligned}
\int_{0}^{\rightarrow \infty} f_{D}(\tau) d \tau & =\alpha ; \\
\alpha f_{D}^{*}(\tau) & =f_{D}(\tau) ; \quad(\tau \text { finite }) . \\
F_{D-}(\tau)=\int_{\tau}^{\infty} f_{D}(\xi) d \xi & =\int_{\tau}^{\rightarrow \infty} f_{D}(\xi) d \xi+(1-\alpha)
\end{aligned}
$$

G. Microb. xv 
where the last term represents the contribution to $F_{D_{-}}(\tau)$ from non-viable organisms; and

$$
\mathscr{C}_{D}(\tau)=2 \alpha e^{-D \tau} f_{D}^{*}(\tau)
$$

It is to be expected that $\alpha$ will diminish with the medium concentration, and so with $D$; this appears to be true in the few known examples which, however, have not yet been investigated systematically. There are also indications that $\alpha$ is much reduced in over-acid media.

\section{Correlation between generation times: its effect on growth rate}

So far we have assumed that the generation time of an organism is independent of its ancestry; this is false, since there is high correlation between the $\tau$ of sisters, and appreciable correlation between the $\tau$ of cousins (and perhaps of second cousins; Powell, 1956b). In experiments lasting for only a short time, the mother-daughter correlation is usually found to be small or zero, but the work of Hughes (1955) suggests that an inherited tendency may persist over many generations. I am not satisfied (Powell, 1956 b) that Hughes's conclusions follow from his results, but they can by no means be rejected at present. We have, then, to estimate the extent to which our previous calculations may be in error because of association between generation times of related organisms. The analysis becomes more difficult, and it will probably be enough to consider the two factors likely to be of major importance: correlation between mothers and daughters, and between sisters.

The following treatment applies to batch cultures; formally, the results for continuous cultures are exactly the same, the only difference being the dependence of the various frequency functions on the substrate concentration, and so on the dilution rate.

Let the product-moment correlation coefficients between generation times be $\rho(H)$ for mothers and daughters, $\rho(S)$ for sisters and let the joint distribution of the generation times of mothers and daughters be $h\left(\tau_{1} ; \tau_{2}\right) d \tau_{1} d \tau_{2}$; this is the probability that the $\tau$ of a mother is in the range $\tau_{1}, \tau_{1}+d \tau_{1}$ and the $\tau$ of a given one of the daughters in $\tau_{2}, \tau_{2}+d \tau_{2}$-we can assume that the two daughters are similarly related to the mother. Then

$$
\int_{0}^{\infty} h\left(\tau_{1} ; \tau\right) d \tau_{1}=\int_{0}^{\infty} h\left(\tau ; \tau_{2}\right) d \tau_{2}=f(\tau)
$$

i.e. the border distributions of $h\left(\tau_{1} ; \tau_{2}\right)$ are alike of the form $f(\tau)$; but $h\left(\tau_{1} ; \tau_{2}\right)$ is not necessarily symmetrical in its arguments, though it appears to be so in practice. Also

$$
\int_{0}^{\infty} \int_{0}^{\infty} \tau_{1} h\left(\tau_{1} ; \tau_{2}\right) d \tau_{1} d \tau_{2}=\int_{0}^{\infty} \int_{0}^{\infty} \tau_{2} h\left(\tau_{1} ; \tau_{2}\right) d \tau_{1} d \tau_{2}=\mathbf{a}
$$

the mean generation time.

We can no longer assume that the distribution of $\tau$ among the organisms present at any given time in the culture is $f(\tau)$; for this "population distribution' we write $\mathscr{P}(\tau)$, and the carrier distribution is

$$
\mathscr{C}(\tau)=2 e^{-\nu_{m} \tau} \mathscr{P}(\tau)
$$

we may also expect $\nu_{m}$ to be different from the value given by (10). 
We deal first with the effect of correlation between sisters. Consider the fissions occurring during a short interval $d t$; these may be represented as in Fig. 5. Each fission is the inception of a pair of sisters, whose generation times, such as $\tau_{21}, \tau_{22}$, may be correlated. Part of the subsequent development of each pair is shown to the right of the interval $d t$. Suppose now the branches of the tree to the right of $t+d t$ are cut off at the points $X$, shuffled in any way, and reattached. We can arrange if we wish that there is no correlation between the members of the pairs formed. But their contribution to the

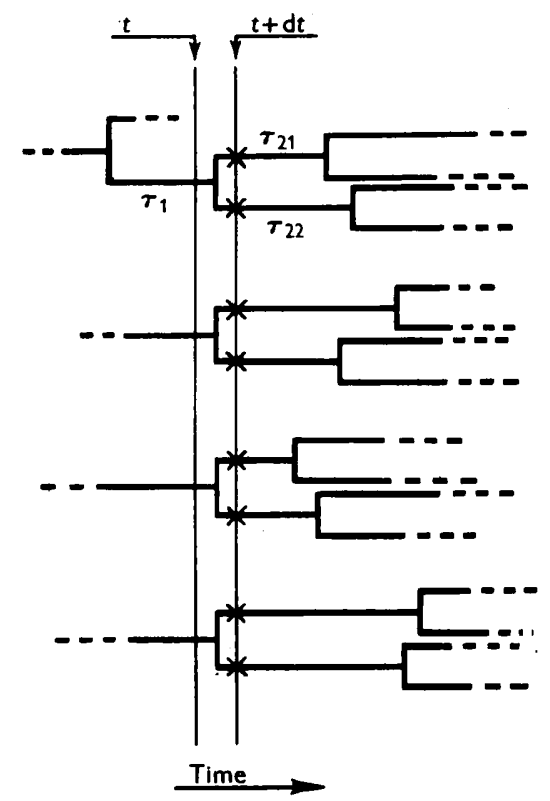

Fig. 5. Illustrating the independence of growth rate and sister-sister correlation. $\tau_{1} ; \tau_{21}, \tau_{22}$ : typical triad of mother and two daughter organisms.

growth rate of the culture remains the same; so for all intervals $d t$. Hence the growth rate is independent of correlation between sisters. This result is true only for steady growth.

Now $\mathscr{P}(\tau)$ must be invariant, i.e. it must be unaltered as members of the population terminate and are replaced by others. Organisms whose generation times are in the range $\tau_{1}, \tau_{1}+d \tau_{1}$ give rise to daughters of which the fraction

$$
\frac{h\left(\tau_{1} ; \tau_{2}\right) d \tau_{1} d \tau_{2}}{f\left(\tau_{1}\right) d \tau_{1}}
$$

have generation times in the range $\tau_{2}, \tau_{2}+d \tau_{2}$. The frequency of such mothers (Fig. 5) is $\mathscr{C}\left(\tau_{1}\right) d \tau_{1}$. The total contribution of daughter organisms to $\mathscr{P}\left(\tau_{2}\right) d \tau_{2}$ from mothers of any $\tau$ is

$$
\int_{0}^{\infty} \mathscr{C}\left(\tau_{1}\right) \frac{h\left(\tau_{1} ; \tau_{2}\right)}{f\left(\tau_{1}\right)} d \tau_{1}=2 \int_{0}^{\infty} e^{-\nu_{m} \tau_{1}} \frac{h\left(\tau_{1} ; \tau_{2}\right)}{f\left(\tau_{1}\right)} \mathscr{P}\left(\tau_{1}\right) d \tau_{1},
$$


and so if $\mathscr{P}(\tau)$ is invariant, it must satisfy

$$
\mathscr{P}(\tau)=2 \int_{0}^{\infty} e^{-\nu_{m} \xi} \frac{h(\xi ; \tau)}{f(\xi)} \mathscr{P}(\xi) d \xi
$$

It is known from the theory of integral equations (see, for example, Whittaker \& Watson, 1940) that

$$
\mathscr{P}(\tau)=\lambda \int \Lambda(\xi ; \tau) \mathscr{P}(\xi) d \xi
$$

has no solution but $\mathscr{P}(\tau) \equiv 0$, unless $\lambda$ has certain special values, the characteristic numbers of the nucleus $\Lambda(\xi ; \tau)$. In our case, $\lambda$ must be 2 , but the nucleus

$$
\Lambda(\xi ; \tau)=e^{-\nu_{m} \xi} \frac{h(\xi ; \tau)}{f(\xi)}
$$

contains a disposable parameter $\nu_{m}$ which provides room for the adjustment of the $\lambda$. Hence from (18) and

$$
\int_{0}^{\infty} \mathscr{P}(\tau) d \tau=\int_{0}^{\infty} \mathscr{C}(\tau) d \tau=2 \int_{0}^{\infty} e^{-\nu_{m} \tau} \mathscr{P}(\tau) d \tau=1
$$

both $\mathscr{P}(\tau)$ and $\nu_{m}$ can in principle be determined. We note that if the $\tau$ of mothers and daughters are statistically independent, $h\left(\tau_{1} ; \tau_{2}\right)=f\left(\tau_{1}\right) f\left(\tau_{2}\right)$ and (18) gives

as it should.

$$
\mathscr{P}(\tau) \equiv f(\tau),
$$

Unfortunately, we have no guidance to the functional form of $h\left(\tau_{1} ; \tau_{2}\right)$. The bivariate normal surface is obviously inappropriate, since both our variables are limited to positive values, and their distribution is markedly skew. The bivariate form of the 'lognormal' distribution (Johnson, 1949) would be acceptable but for its intractability in the present context, and the distributions derived by Narumi $(1923 a, b)$ are unsuitable for other reasons as well. In the following examples I have used a purely numerical method to obtain estimates of $\mathscr{P}(\tau)$ in histogram form, from observed frequencies of $f(\tau)$ and $h\left(\tau_{1} ; \tau_{2}\right)$; but I have not been able to prove that the iterative process converges, though it appears to do so from both sides of the apparent limit.

\section{Examples}

(i) (Data from Powell, 1956b.) Chromobacterium prodigiosum growing on tryptic meat broth at $35^{\circ}$.

$$
\begin{aligned}
\mathbf{a} & =\mathbf{2 2 \cdot 8 7} \mathrm{min} ., \\
\operatorname{var} \tau & =\mathbf{2 3 \cdot 3 4}, \\
c & =\mathbf{0} \cdot 211 .
\end{aligned}
$$

Growth rate, assuming $\rho(H)=0$,

estimated from Type III fitted function: $\nu_{m}=0 \cdot 03075$, estimated directly from the observations: $\nu_{m}=0.03075$,

Estimated $\rho(H)=+0.302$.

$(\log 2) / \mathbf{a}=0 \cdot 03030$.

Estimated $\rho(S)=+0 \cdot 698$. 
The correlation table of the observations representing $h\left(\tau_{1} ; \tau_{2}\right)$ was slightly unsymmetrical, and the border distributions had slightly (but not significantly) different means; Bowker's (1948) test showed that the lack of symmetry was not significant. To ensure that the border distributions were exactly alike, a new table was constructed, in which the entries were

$$
h_{s}\left(\tau_{1} ; \tau_{2}\right)=\frac{1}{2}\left\{h_{0}\left(\tau_{1} ; \tau_{2}\right)+h_{0}\left(\tau_{2} ; \tau_{1}\right)\right\} \text {, }
$$

where $h_{0}\left(\tau_{1} ; \tau_{2}\right)$ is the observed frequency in the cell $\tau_{1} ; \tau_{2}$. This manipulation of the data involved little disturbance, and the estimated $\rho(H)$ for the new table was 0.299 , differing from the original by much less than the sampling error. The new border frequencies were called $f_{s}(\tau)$ and their sum was called $W$. An approximation to the solution of (18) was obtained by carrying out the following scheme of iteration:

$$
\left\{\begin{aligned}
\boldsymbol{P}_{r+1}^{\prime}(\tau) & =\sum_{\xi} e^{-\nu_{0} \xi} \frac{h_{s}(\xi: \tau)}{f_{s}(\xi)} P_{r}(\xi) \\
\lambda_{r+1} & =W / \sum_{\tau} P_{r+1}^{\prime}(\tau) \\
P_{r+1}(\tau) & =\lambda_{r+1} P_{r+1}^{\prime}(\tau)
\end{aligned}\right.
$$

For the first step, the set $\boldsymbol{P}_{0}(\xi)$ was taken as $f_{s}(\xi)$, and $\nu_{0}$ was taken as $\mathbf{0 . 0 8 0 7 5}$, on the assumption that $\mathscr{P}(\tau)$ did not differ much from $f(\tau)$. After 8 cycles, the set $P(\tau)$ no longer changed, and $\lambda$ was constant at 1.9913. The iteration was then repeated with better approximations to $\nu_{m}$ than $\nu_{0}$. If in the integral equation

$$
\boldsymbol{P}(\tau)=\lambda \int_{0}^{\infty} e^{-\nu \xi} \frac{h(\xi ; \tau)}{f(\xi)} \boldsymbol{P}(\xi) d \xi
$$

$P$ is a frequency function, and we take $\nu=0$, we have $\lambda=1$, for

$$
\mathbf{1}=\int_{0}^{\infty} P(\tau) d \tau=\int_{0}^{\infty} \lambda \int_{0}^{\infty} \frac{h(\xi ; \tau)}{f(\xi)} P(\xi) d \xi d \tau=\lambda \int_{0}^{\infty} P(\xi) d \xi=\lambda .
$$

But if $\nu=\nu_{m}, \lambda=2$. Hence if we finally obtain by iteration a constant $\lambda=\lambda_{\infty}$ with $\nu=v_{0}$, we might expect

$$
\nu_{1}=\frac{v_{0}}{\lambda_{\infty}-1}
$$

to be a better approximation to $\nu_{m}$. At the third trial, it was found that

$$
v_{2}=0.03130
$$

gave $\lambda=1.9994$, and this was considered sufficiently accurate. Approximately, therefore, the growth rate is

$$
v_{m}=0.0313 \mathrm{~min} .^{-1} \text {, }
$$

about $2 \%$ greater than the value obtained by disregarding the motherdaughter correlation. The final set of $\boldsymbol{P}(\tau)$, approximating to $\mathscr{P}(\tau)$, was used to construct the histograms in Fig. $6 a, b$.

(ii) (Data from Powell, 1956b.) Bacterium (Aerobacter) cloacae growing. on tryptic meat broth at $35^{\circ}$.

$$
\begin{aligned}
\mathbf{a} & =\mathbf{2 4} \cdot 43, \\
\operatorname{var} \tau & =26 \cdot 92, \\
c & =0 \cdot 212 .
\end{aligned}
$$


Growth rate, assuming $\rho(H)=0$,

estimated from Type III fitted function: $\nu_{m}=\mathbf{0} \cdot \mathbf{0 2 8 8 6}$, estimated directly from the observations: $\nu_{m}=0.02884$,

Estimated $\rho(H)=-0 \cdot 316$.

$(\log 2) / \mathbf{a}=0 \cdot 02838$.

Estimated $\rho(S)=+0 \cdot 603$.

By the same procedure as in example (i) it was found that

$$
\nu_{m}=\mathbf{0 . 0 2 8 1 7} \text {. }
$$

This is about $2 \frac{1}{2} \%$ less than the value to be expected in the absence of correlation and in fact less than $(\log 2) / \mathbf{a}$.
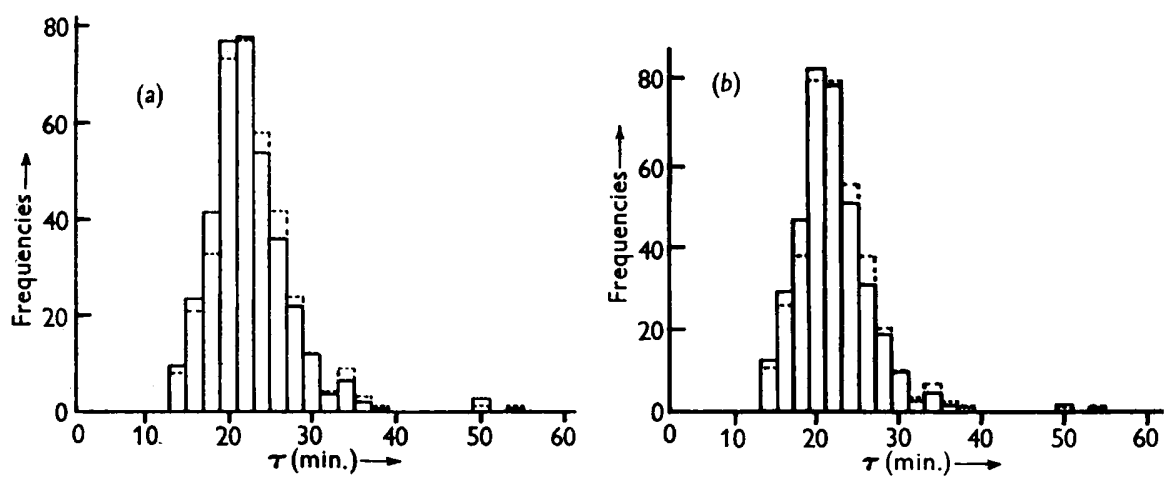

Fig. 6. (a) Chromobacterium prodigiosum. Continuous line histogram: population distribution, calculated from generation time distribution and mother-daughter correlation. Dotted: observed generation time distribution. (b) C. prodigiosum. Continuous line histogram : carrier distribution, calculated from generation time distribution and motherdaughter correlation. Dotted: carrier distribution expected in the absence of motherdaughter correlation.

These two examples were chosen out of eighteen available because the sample values of $\rho(H)$ were extreme. Most of the $\rho(H)$ were much smaller, the average being slightly positive. As might be expected, positive $\rho(H)$ tends to increase $\nu_{m}$ for a given $f(\tau)$, negative $\rho(H)$ to diminish it. But the productmoment coefficient is not really an appropriate numerical measure of the kind of correlation which is effective in making $\mathscr{P}(\tau)$ differ from $f(\tau)$-the first product-moment itself can be zero even if $h\left(\tau_{1} ; \tau_{2}\right) \neq f\left(\tau_{1}\right) f\left(\tau_{2}\right)$.

It may be added that (18) (with $D$ for $\nu_{m}, f_{D}(\tau)$ for $f(\tau)$ etc.) applies equally to continuous cultures.

I conclude that the effect of mother-daughter correlation on growth rate can be neglected for the time being; that equation (10) gives the relation between $\nu_{m}$ and $f(\tau)$ with sufficient accuracy for present needs. However, it cannot yet be said that correlation does not sensibly affect the selective power of the system; we do not know that $\mathscr{P}(\tau)$ and $f(\tau)$ are of the same order of magnitude for fairly large $\tau$. It is still possible, moreover, that grandmothergranddaughter (or higher order) correlation is not negligible. 


\section{COMMENT}

The foregoing paragraphs are mainly intended to provide part of a theoretical background for the detailed study of bacterial growth in continuous cultures, and do not in themselves call for further discussion. It is worth while, however, to consider the probable range of validity of the principal assumptions.

(i) In all actual cultures the number of their proper organisms is so large that the assumption of (mathematical) continuity and determinate behaviour cannot lead to false results. Strictly interpreted, equation (16), for example, is an assertion about expectations only-there is a finite though exceedingly small chance that all the organisms might be washed out of a continuous culture vessel under conditions favourable to continued growth. In fact the culture is self-regulating, and any accidental change in population density is compensated by a changed growth rate which tends to restore the equilibrium. Where the future of the culture turns on the presence of a few organisms (e.g. mutants) only, a deterministic treatment is no longer adequate.

(ii) The steady growth of a batch culture can be maintained for a relatively short time only. However, some calculations by Dr W. A. O'N. Waugh (private communication) show that a few generations after the end of the lag phase are enough to produce an unchanging age distribution in the population. On the other hand, the duration of a continuous culture has no definite limit, and may chance to be prolonged for months (see, for example, Herbert et al. 1956). In uncomplicated cases there is then no doubt that a steady state is reached and maintained.

(iii) The assumption of 'perfect' mixing - of instantaneous and homogeneous dispersal of the ingoing medium-greatly simplifies the theoretical and experimental analysis of continuous cultures. It is obvious that for purposes of study, at least, the degree of mixing should be well defined in some sense, but it is not at all obvious that an adequate approximation to 'perfect' mixing is practically attainable. The exigencies of the assumption can be appreciated thus: let a closed surface be imagined to be drawn anywhere within the culture fluid. Then the rate at which fresh medium enters this surface must bear a constant ratio to the volume enclosed by it, no matter what its shape or disposition. A continuous culture is particularly sensitive to lack of homogeneity, because of the steep fall in output near the critical dilution rate. Moreover, the growth rate is not strictly equal to the dilution rate unless the mixing is perfect. This subject needs greater attention than has hitherto been given it, and cannot properly be discussed here. Some experiments of my own, not yet published, suggest that mixing can be made sensibly perfect in laboratory apparatus up to a few litres in capacity.

(iv) Two assumptions commonly made in treating continuous cultures, but not made explicit above are: (a) that growth rate responds instantly to changes in medium concentration; (b) that growth is not inhibited or promoted by products of metabolism or by imbalance in the medium caused by the organisms themselves. Failure of either assumption can demonstrably lead to sustained oscillations in population density. 


\section{SYNOPSIS}

Here the principal results are summarized without reference to the limitations imposed by the assumptions made.

(i) The growth rate $(\nu)$ of a culture is not simply related to the generation time distribution. In batch cultures, when $v$ has nearly its maximum value $\nu_{m}$, the relation is given by equation (10), where $f(\tau)$ is the frequency function of generation time. In continuous cultures $\nu$ is equal to the dilution rate $D$. Since $D<\nu_{m}$, growth is restricted; the frequency function must alter, in scale if not in shape, so as to satisfy equation (16).

(ii) The frequency function of age $(\phi(a))$ is always a J-shaped curve with a finite maximum at $a=0$; the younger organisms are present in the greater numbers. Its dependence on the generation time distribution is given by equations (9) and (15).

(iii) It is convenient to distinguish two different generation time distributions in a growing culture. The population distribution, $\mathscr{P}(\tau)$, is the distribution for all organisms present at any one instant. When there is negligible correlation between the generation times of related organisms, $\mathscr{P}(\tau) \equiv f(\tau)$. The mothers of the organisms present at any one time have a different generation time distribution, the carrier distribution, $\mathscr{C}(\tau)$. In continuous cultures, some of the organisms present at any one time will be washed out before they divide. Those which remain in the vessel until their termination have the distribution $\mathscr{C}(\tau)$. Equations (11) and (17) determine $\mathscr{C}(\tau)$ in terms of $f(\tau)$.

(iv) Correlation between the generation times of sister organisms does not affect the growth rate. Positive mother-daughter correlation increases it, negative diminishes it. The population distribution depends on the joint distribution of generation times of mothers and daughters according to equation (18), and is not then equal to $f(\tau)$ in general. The available data suggest that mother-daughter correlation is small enough to be neglected for the time being. If it has to be taken into account, $f(\tau)$ must be replaced by $\mathscr{P}(\tau)$ (as determined by (18)) in expressions for $\nu, \phi(a)$ and $\mathscr{C}(\tau)$.

I am indebted to Dr D. Herbert for some suggestions and criticism.

\section{REFERENCES}

Bowker, A. M. (1948). A test for symmetry in contingency tables. J. Amer. statist. Ass. 43, 572.

Bryson, V. \& Szybalski, W. (1952). Microbial selection. Science, 116, 45.

Dagley, S. \& Hinshelwood, C. N. (1938). Physico-chemical aspects of bacterial growth. Part I. Dependence of growth of Bact. lactis aerogenes on concentration of medium. J. chem. Soc. p. 1930.

Feluer, W. (1950). Probability Theory and its Applications. New York: John Wiley and Sons.

HarRIs, T. E. (1951). Some mathematical models for branching processes. Proc. Second Berkeley Symposium on Mathematical Statistics and Probability, p. 305. 
Herbert, D., Elsworth, R. \& Teluing, R. C. (1956). The continuous culture of bacteria: a theoretical and experimental study. J. gen. Microbiol. 14, 601.

Hughes, W. H. (1955). The inheritance of differences in growth rate in Escherichia coli. J. gen. Microbiol. 12, 265.

Jonnson, N. L. (1949). Bivariate distributions based on simple translation systems. Biometrika, 36, 297.

KEndall, D. G. (1948). On the role of variable generation time in the development of a stochastic birth process. Biometrika, 35, 316.

KENDALL, D. G. (1952). On the choice of a mathematical model to represent normal bacterial growth. J. R. statist. Soc. B, 14, 41.

Monod, J. (1942). Recherches sur la croissance des cultures bactériennes. Paris: Hermann et Cie.

Monod, J. (1950). La technique de culture continue, théorie et applications. Ann. Inst. Pasteur, 79, 390.

NARUMI, S. (1923 $a$ ). On the general forms of bivariate frequency distributions which are mathematically possible when regression and variation are subjected to limiting conditions. Part I. Biometrika, 15, 77.

Narumi, S. (1923b). On the general forms of bivariate frequency distributions which are mathematically possible when regression and variation are subjected to limiting conditions. Part II. Biometrika, 15, 209.

Novick, A. (1955). Growth of bacteria. Annu. Rev. Microbiol. 9, 97.

Novick, A. \& SzIlard, L. (1950). Description of the chemostat. Science, 112, 715.

Pearson, E. S. \& Hartey, H. O. (1954). Biometrika Tables for Statisticians, vol. I. Cambridge University Press.

Powel., E. O. (1955). Some features of the generation times of individual bacteria. Biometrika, 42, 16.

Powell, E. O. (1956a). A rapid method for determining the proportion of viable bacteria in a culture. J. gen. Microbiol. 14, 153.

Powell, E. O. (1956 b). The pattern of bacterial generation times. Biometrika, 43, (In the Press.)

Rahn, O. (1932). A chemical explanation of the variability of the growth rate. J. gen. Physiol. 15, 257.

Topley \& Wilson's Principles of Bacteriology and Immunity (1955). 4th edition, ed. by Wilson, G. S. \& Miles, A. A. London: Edward Arnold.

Whittaker, E. T. \& Watson, G. N. (1940). Modern Analysis, 4th ed. Cambridge University Press. 